\title{
TGF- $\beta$ Signalling is Suppressed under Pro-Hypertrophic Conditions in MSC Chondrogenesis Due to TGF- $\beta$ Receptor Downregulation
}

\author{
Christian G. Pfeifer ${ }^{1,2}$, Alexandra Karl ${ }^{1}$, Maximilian Kerschbaum ${ }^{1,2}$, Arne Berner ${ }^{1}$, Siegmund Lang ${ }^{1,2}$, \\ Rupert Schupfner, ${ }^{3}$ Matthias Koch ${ }^{1,2}$, Peter Angele ${ }^{1,2}$, Michael Nerlich ${ }^{1,2}$, Michael B. Mueller ${ }^{1,2,3}$ \\ ${ }^{1}$ Laboratory of Experimental Trauma Surgery, Department of Trauma Surgery, University Regensburg Medical Centre, Regensburg, Germany \\ ${ }^{2}$ Department of Trauma Surgery, University Regensburg Medical Centre, Regensburg, Germany \\ ${ }^{3}$ Department of Trauma and Reconstructive Surgery, Klinikum Bayreuth, Bayreuth, Germany
}

Background and Objectives: Mesenchymal stem cells (MSCs) become hypertrophic in long term despite chondrogenic differentiation following the pathway of growth plate chondrocytes. This terminal differentiation leads to phenotypically unstable cartilage and was mirrored in vitro by addition of hypertrophy inducing medium. We investigated how intrinsic TGF- $\beta$ signaling is altered in pro-hypertrophic conditions.

Methods and Results: Human bone marrow derived MSC were chondrogenically differentiated in 3D culture. At day 14 medium conditions were changed to 1 . pro-hypertrophic by addition of T3 and withdrawal of TGF- $\beta$ and dexamethasone 2. pro-hypertrophic by addition of BMP 4 and withdrawal of TGF- $\beta$ and dexamethasone and 3. kept in prochondrogenic medium conditions. All groups were treated with and without TGF $\beta$-type-1-receptor inhibitor SB431542 from day 14 on. Aggregates were harvested for histo- and immunohistological analysis at d14 and d28, for gene expression analysis (rt-PCR) on $\mathrm{d} 1, \mathrm{~d} 3, \mathrm{~d} 7, \mathrm{~d} 14, \mathrm{~d} 17, \mathrm{~d} 21$ and $\mathrm{d} 28$ and for Western blot analysis on $\mathrm{d} 21$ and d28. Induction of hypertrophy was achieved in the pro-hypertrophic groups while expression of TGF $\beta$-type-1- and 2-receptor and Sox 9 were significantly downregulated compared to pro-chondrogenic conditions. Western blotting showed reduced phosphorylation of Smad 2 and 3 in hypertrophic samples, reduced TGF- $\beta-1$ receptor proteins and reduced SOX 9. Addition of SB431542 did not initiate hypertrophy under pro-chondrogenic conditions, but was capable of enhancing hypertrophy when applied simultaneously with BMP-4.

Conclusions: Our results suggest that the enhancement of hypertrophy in this model is a result of both activation of pro-hypertrophic BMP signaling and reduction of anti-hypertrophic TGF $\beta$ signaling.

Keywords: Mesenchymal stem cells, Chondrogenesis, Terminal differentiation, Receptor

Received: September 27, 2018, Revised: December 21, 2018, Accepted: December 28, 2018, Published online: February 28, 2019 Correspondence to Christian G. Pfeifer

Department of Trauma Surgery, University Regensburg Medical Centre, Franz-Josef-Strauss-Allee 11, Regensburg 93053, Germany Tel: +49-941-944-6792, Fax: +49-941-944-6806

E-mail: christian.pfeifer@ukr.de

(c) This is an open-access article distributed under the terms of the Creative Commons Attribution Non-Commercial License (http://creativecommons.org/ licenses/by-nc/4.0/), which permits unrestricted non-commercial use, distribution, and reproduction in any medium, provided the original work is properly cited.

Copyright (c) 2019 by the Korean Society for Stem Cell Research

\section{Introduction}

The repair capacity of articular cartilage after injury or disease is poor. Mesenchymal stem cells are promising candidates for the use of cell based tissue engineering applications for the repair of cartilage tissue lesions. The chondrogenic potential of MSCs has been shown in different matrix free and matrix based cell culture systems (1-7). In the commonly used pellet culture system, MSCs differentiate chondrogenically in a serum free medium containing TGF $\beta$ and dexamethasone (6). However, chon- 
drogenic differentiating MSCs express hypertrophy markers like collagen type $\mathrm{X}$, alkaline phosphatase (ALP) and MMP-13 (8-11). This behaviour of chondrogenic differentiating MSCs resembles that of growth plate chondrocytes during endochondral ossification. During endochondral bone development growth plate chondrocytes do not rest on a developmental stage typical for articular chondrocytes but spontaneously proceed towards the hypertrophic stage. Hypertrophic chondrocytes then undergo apoptosis, the tissue is invaded by blood vessels and osteoprogenitor cells and bone is formed. Vascular invasion and matrix calcification has also been observed after in vivo transplantation of human chondrogenic MSC pellet cultures into mice $(12,13)$. This biological behaviour of chondrogenic differentiating MSCs raises concern for a tissue engineering application of MSCs in articular cartilage repair. It is important to better understand the mechanisms that regulate late differentiation steps in chondrogenic differentiating MSCs to find ways to inhibit hypertrophy. The similarity of MSC chondrogenesis and embryonic endochondral ossification indicates that similar mechanisms are involved in both biological processes (10).

TGF $\beta$ signaling has been shown to play a crucial role in the regulation of endochondral ossification. In vivo and in vitro studies showed that TGF $\beta$ signaling promotes chondrogenic differentiation of mesenchymal cells and embryonic chondrocytes (14-18). In addition, TGF $\beta$ signaling is important in the regulation of chondrocyte maturation. TGF $\beta$ signaling inhibits hypertrophy in vitro and in vivo. In vitro studies showed that TGF $\beta$ inhibits hypertrophy and the expression of hypertrophic markers like collagen type X and ALP in cultured embryonic chondrocytes (19-21). In vivo it was shown that the application of TGF $\beta$ into a developing chick limb inhibits chondrocyte hypertrophy and loss of function models of TGF $\beta$ signaling result in premature chondrocyte hypertrophy in mice (22-24).

$\operatorname{TGF} \beta 1,2$ and 3 are the classical inducers of MSC chondrogenesis $(1,7)$. However, the exact role of TGF $\beta$ signaling in the regulation of MSC hypertrophy is relatively unknown. Here we used an in vitro hypertrophy model for chondrogenic differentiating MSCs in which the hypertrophic phenotype can be strongly enhanced by modulations in the medium conditions. Differential expression analysis of TGF $\beta$ signaling associated genes was carried out between standard chondrogenic and hypertrophy enhancing conditions, TGF $\beta$ signaling activity was measured comparatively between the two conditions and functional experiments using TGF $\beta$ signaling modulators were conducted.

\section{Materials and Methods}

\section{Isolation of MSCs}

MSCs were isolated from iliac crest bone marrow aspirates of seven male patients, aged 21 to 42 years, undergoing surgery that required autologous bone grafting with approval of the local ethics committee and informed written consent. MSCs were isolated by Ficoll (Biochrom) gradient centrifugation followed by plastic adhesion. Cells were expanded in Dulbecco's modified Eagle's medium (DMEM) low glucose (Invitrogen) with $10 \%$ fetal calf serum (PAN Biotech $\mathrm{GmbH}$ ) and $1 \%$ penicillin/streptomycin (Invitrogen) at $37^{\circ} \mathrm{C}$ with $5 \% \mathrm{CO}_{2}$. The medium was changed twice a week and cells were trypsinized at $80 \%$ confluence and frozen for later use in liquid nitrogen. After thawing and monolayer expansion, cells were used for the experiments at passage 1 .

\section{Chondrogenic differentiation and enhancement of hypertrophy}

MSCs were trypsinized and seeded in V-bottomed 96-well polypropylene plates at 200,000 cells per well. Pellets were formed by centrifugation at $250 \mathrm{~g}$ for $5 \mathrm{~min}$ and chondrogenically differentiated in DMEM with high glucose (Invitrogen), 1\% ITS (Sigma Aldrich), $50 \mu \mathrm{g} / \mathrm{ml}$ ascorbate-2-phosphate (Sigma Aldrich), $40 \mu \mathrm{g} / \mathrm{ml} \mathrm{L-pro-}$ line (Sigma Aldrich), $100 \mathrm{nM}$ dexamethasone (Sigma Aldrich), $1 \mathrm{mM}$ sodium pyruvat (Invitrogen) and $10 \mathrm{ng} / \mathrm{ml}$ TGF $\beta 1$ (R\&D Systems).

After a pre-differentiation period of 14 days, medium conditions were changed to hypertrophy enhancing medium (hyp) consisting of DMEM high glucose, 1\% ITS, $50 \mathrm{~g} / \mathrm{ml}$ ascorbate-2-phosphate, $40 \mathrm{~g} / \mathrm{ml} \mathrm{L}$-proline, $1 \mathrm{nM}$ triiodothyronine (T3) (Sigma Aldrich) and the control was kept in chondrogenic medium (chon) for the whole culture period. The medium was changed three times per week.

Aggregates were harvested at d1, d3, d7, d14, d17, d21 and $\mathrm{d} 28$ for gene expression analysis. Aggregates for histological analysis were harvested on $\mathrm{d} 14$ and $\mathrm{d} 28$.

\section{Modulation of hypertrophy by TGF $\beta$-type-1-receptor inhibitor SB431542}

Aggregates were differentiated chondrogenically for 14 days. On day 14, aggregates were either kept in chondrogenic medium with $0.5 \mu \mathrm{M}, 3 \mu \mathrm{M}$ or $10 \mu \mathrm{M} \mathrm{SB} 431542$ (Tocris) or transferred to hypertrophic medium with $0.5 \mu \mathrm{M}$, $3 \mu \mathrm{M}$ or $10 \mu \mathrm{M}$ SB431542 for additional 14 days. Aggregates that were kept in chondrogenic and hypertrophic medium without SB431542 were used as a control. 
Table 1. Primer sequences for real time PCR

\begin{tabular}{lll}
\hline \multicolumn{1}{c}{ Gene } & \multicolumn{1}{c}{ Sequence (forward) } & \multicolumn{1}{c}{ Sequence (reverse) } \\
HPRT & CGAGATGTGATGAAGGAGATGG & GCAGGTCAGCAAAGAATTTATAGC \\
Sox9 & ACACACAGCTCACTCGACCTTG & AGGGAATTCTGGTTGGTCCTCT \\
TGF $\beta$ R1 & GAACCTGCTCTCCTGCTTG & GCCTCATCTGCTCAATCTCC \\
TGF $\beta$ R2 & GGAAACTTGACTGCACCGT & CTGCACATCGTCCTGTGG \\
TGF $\beta 1$ & ACATCAACGGGTTCACTAC & CTGAAGCAATAGTTGGTGTCC \\
TGF $\beta 3$ & ACACACAAGCAACAAACCTCAC & AACCAAACCCACACTTTCTTTACC \\
\hline
\end{tabular}

In another experiment aggregates were pre-differentiated in chondrogenic medium for 14 days followed by incubation in chondrogenic medium including $100 \mathrm{ng} / \mathrm{ml}$ BMP4 (R\&D systems) and $0.5 \mu \mathrm{M}, 3 \mu \mathrm{M}$ or $10 \mu \mathrm{M}$ SB431542.

Aggregates were histologically investigated on $\mathrm{d} 28$.

\section{Histology, histochemistry and immunohistochemistry}

Aggregates were harvested on d14 and d28, fixed in 4\% paraformaldehyde and $10 \mu \mathrm{m}$ thick frozen sections were prepared.

Sections were stained with dimethylmethylene blue (DMMB) (Sigma Aldrich) for glycosaminoglycans. Histochemical ALP staining was performed with an alkaline phosphatase kit (Sigma Aldrich) with neutral red as counterstain.

For immunohistochemistry mouse anti collagen type $\mathrm{X}$ (1 : 20, Quartett Immunodiagnostika und Biotechnologie $\mathrm{GmbH})$ and mouse anti collagen type II (1 : 100, Calbiochem) antibodies were used and immunohistochemistry was carried out as follows: . After blocking of endogenous peptidases $\left(3 \% \mathrm{H}_{2} \mathrm{O}_{2} / 10 \%\right.$ Methanol in PBS) for $30 \mathrm{mi}$ nutes, antigen retrieval with pepsin digestion for $15 \mathrm{mi}$ nutes at room temperature (RT) was performed. Then sections were incubated in blocking buffer $(10 \%$ fetal bovine serum $/ 10 \%$ goat serum in PBS) for 60 minutes at RT followed by incubation in an appropriate primary antibody in blocking buffer overnight at $4^{\circ} \mathrm{C}$. For collagen type $\mathrm{X}$ staining additional hyaluronidase digestion for 60 minutes at RT was performed prior to blocking. Immunolabeling was detected with a biotinylated secondary antibody (1 : 100; Dianova), horseradish peroxidase conjugated streptavidin (Vector Laboratories, Burlingame) and metal enhanced diaminobenzidine as substrate (Sigma Aldrich).

\section{RNA isolation, cDNA synthesis and gene expression analysis}

For each of the 7 different independent donors 8 to 10 aggregates per condition and time point were pooled for the experiments, homogenized in $1 \mathrm{ml}$ TRI Reagent
(Sigmal Aldrich) using the Power Gen 1000 homogenizer (Fisher Scientific) and RNA was isolated by the Trizol method. Reverse transcription was performed with Transcriptor First Strand cDNA Synthesis kit (Roche). Semiquantitative real-time PCR was performed with Brilliant SYBR Green QPCR mix (Stratagene) and the Mx3000P QPCR System (Stratagene). Gene expression was normalized to hypoxanthine guanine phosphoribosyltransferase (HPRT).

For primers use din real time PCR please see Table 1.

\section{Western blot analysis}

For Western Blot Analysis, the following antibodies were used: mouse anti Sox9 (1 : 500, Millipore); rabbit anti TGF $\beta$ R1 (1:500, Abcam); rabbit anti $\beta$ actin (1: 10000, Abcam); rabbit anti Smad2 (1:1000, Cell Signaling); rabbit anti Smad3 (1 : 1000, Cell Signaling); rabbit anti phospho-Smad2 (1 : 1000, Cell Signaling); rabbit anti phospho-Smad3 (1 : 1000, Cell Signaling).

5 to 8 MSC pellets per time point and per condition for each patient were pooled, washed in ice cold PBS and homogenized in $500 \mu 16 \mathrm{M}$ urea/2\% SDS solution containing protease inhibitor cocktail (Sigma) and phosphatase inhibitor (Sigma) using the Power Gen 1000 homogenizator (Fischer Scientific). The lysate was centrifuged for 5 minutes at $1000 \mathrm{~g}\left(4^{\circ} \mathrm{C}\right)$ and the supernatant was transferred to a fresh tube. The protein concentration of the supernatant was determined using the BCA Protein Assay kit (Biorad, DC Protein Assay) according to the manufacturer's instructions.

Lysates were supplemented with $4 \times$ LDS sample buffer (Invitrogen) and $10 \mathrm{mM}$ dithiothreitol (DTT) and proteins were denatured for 5 minutes at $95^{\circ} \mathrm{C}$. For gel electrophoresis, equal amounts of protein $(10 \mu \mathrm{g})$ were loaded and separated on a $4 \sim 12 \%$ bis tris gel (Novex by Life Technologies) at $120 \mathrm{~V}$. After gel electrophoresis proteins were transferred from the gel to a polyvinylidenfluoride (PVDF) membrane (Millipore). Blotting was performed for 2 hours at $100 \mathrm{~V}$. After transfer, the membrane was blocked for 1 hour in 5\% skim milk powder in TRIS buf- 
fered saline with Tween 20 (TBST). The membrane was then incubated in primary antibody in $5 \%$ skim milk powder in TBST over night at $4^{\circ} \mathrm{C}$. The next day, the membrane was washed three times for 10 minutes in TBST and afterwards incubated in HPR-coupled secondary antibody ( $1: 1000$, Pierce) in 5\% skim milk powder in TBST at room temperature for 1 hour. Then the membrane was washed three times in TBST for 10 minutes. Chemoluminescence was detected with the ECL western kit (Pierce) and by using x-ray sensitive films (ECL Hyperfilm, Amersham). The films were developed in a photo developer (Curix 60,) AGFA).

Western Blot membranes were stripped using Re-blot Plus (Millipore) according to manufactures instructions.

\section{Statistical analysis}

Gene expression was analyzed by calculating the means of every relative expression normalized to the housekeeping gene HPRT. After checking for normal distribution by Kolmogorow-Smirnoff testing a two tailed student's t-test was used. To maintain an overall p-level of $\mathrm{p}<0.05$, posthoc Bonferronis testing was carried out.

\section{Results}

\section{Enhancement of hypertrophy}

In our study the enhancement of hypertrophy by addition of T3 was shown by an increased cell size, stronger collagen type $\mathrm{X}$ staining and higher ALP activity under pro-hypertrophic conditions compared to standard chondrogenic conditions. Extracellular matrix analysis revealed poorer glycosaminoglycan content (by DMMB staining, Fig. 1A, B), and immunohistochemically equal to less collagen type 2 staining (Fig. 1C, D) after hypertrophic conversion. Analysis for hypertrophic markers showed increased collagen type 10 staining (Fig. 1E, F) as well as strongly increased staining for the pre-apoptotic marker alkaline phospathase (Fig. 1G, H) for samples after hypertrophic conversion compared to non-converted samples.

\section{TGF $\beta$ signaling activity is reduced under hypertrophic conditions}

We detected a significant down-regulation of TGF $\beta$ receptor expression under hypertrophy enhancing conditions. Real time PCR revealed that the TGF $\beta$ receptor 1 (TGF $\beta$ R1) is significantly down-regulated under hypertrophic conditions on day 17, day 21 and day 28 compared
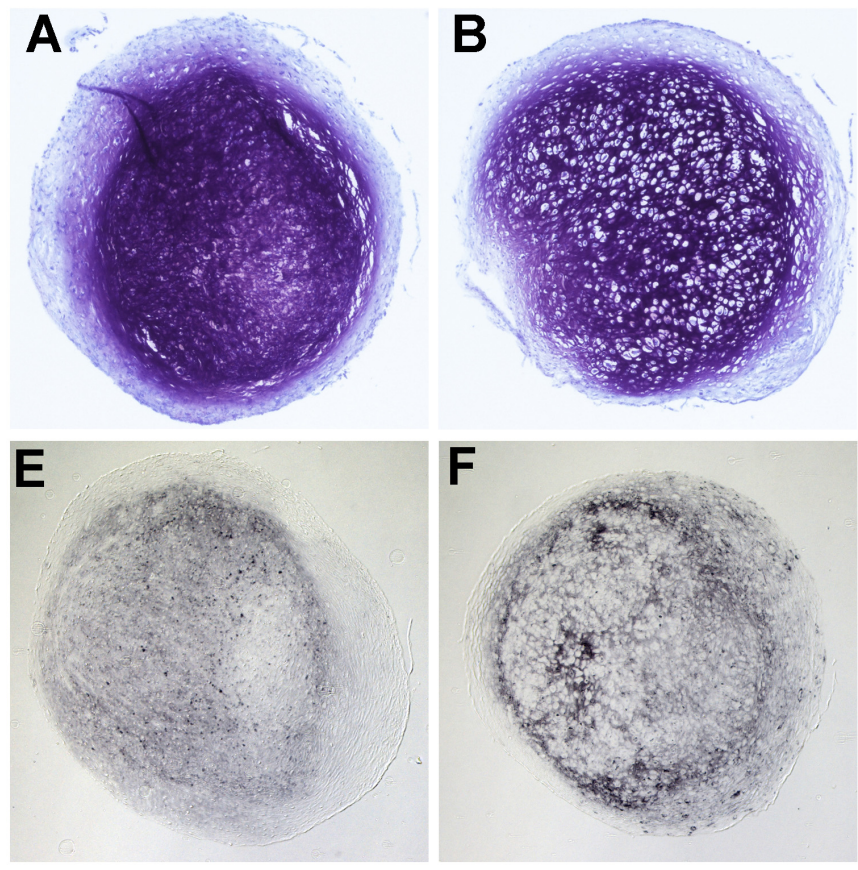
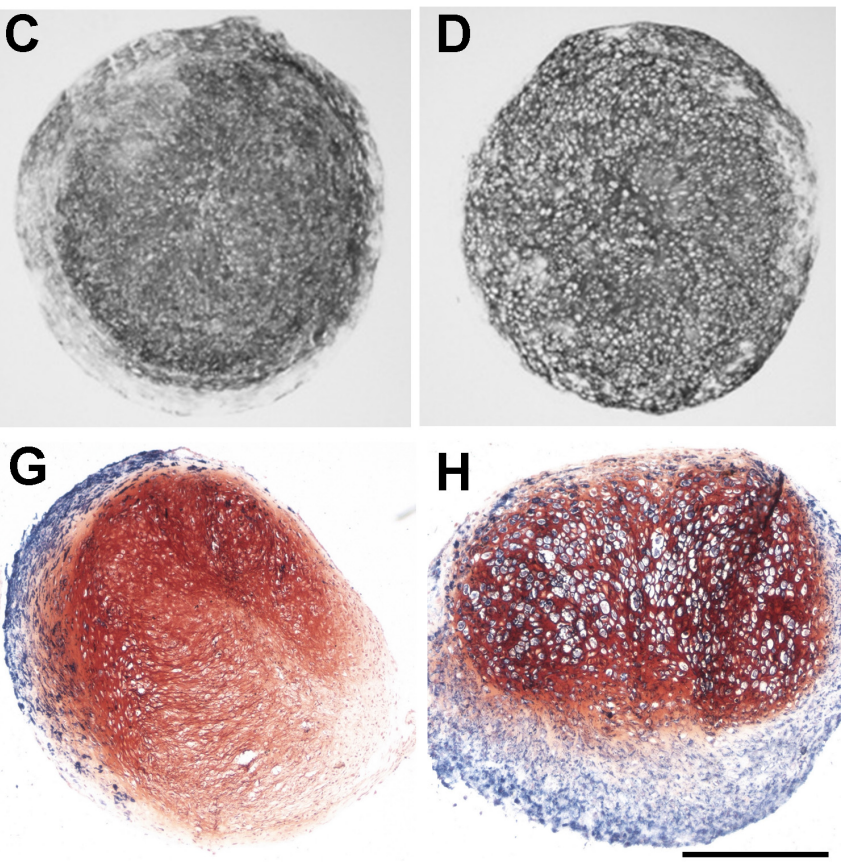

Fig. 1. Differences in glycosaminoglycan content shown by DMMB staining of cell pellets after chondrogenic (A) and hypertrophic conditions (B), as well as different collagen 2 production as revealed by immunohistochemistry against collagen 2 of cell pellets after chondrogenic (C) and hypertrophic conditions (D). Enhancement of hypertrophy shown by immunohistochemistry against collagen 10 between chondrogenic (E) and hypertrophic (F) conditioned cell pellets as well as by ALP staining of chondrogenic $(\mathrm{G})$ and hypertrophic $(\mathrm{H})$ conditioned cell pellets (Scale bar $=500 \mu \mathrm{m}$ ). 
A

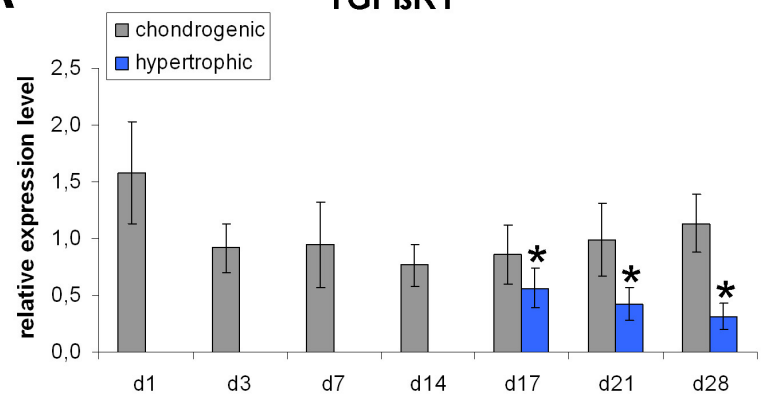

C

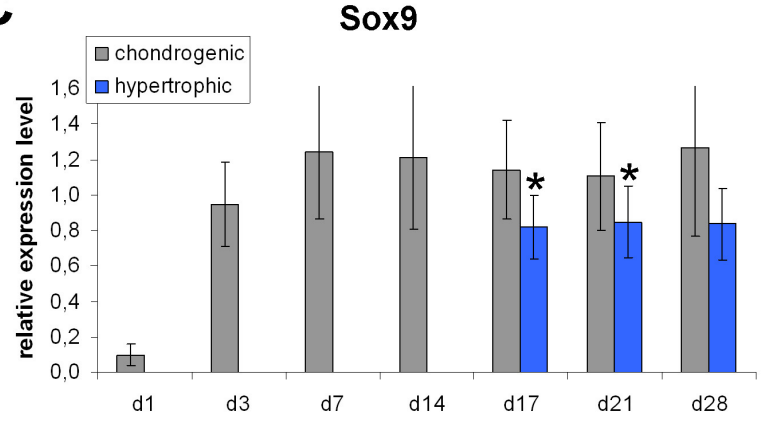

to chondrogenic conditions (Fig. 2A). The TGF $\beta$ receptor 2 (TGF $\beta$ R2) is significantly down-regulated on day 28 in hypertrophic MSC pellets (Fig. 2B). On protein level, Western Blot analysis detected a decreased amount of TGF $\beta$ R1 protein under hypertrophic conditions on day 21 and day 28 compared to chondrogenic conditions (Fig. $4 \mathrm{~A})$. The amount of Sox 9 protein is reduced on day 21 under hypertrophic conditions but not on day 28 (Fig. $4 \mathrm{~B})$. Western blotting thus reinforced our rtPCR results and revealed again suppression of TGF $\beta$ receptors 1 and 2 expression as well as Sox 9 release under hypertrophic conditions.

In order to investigate, whether there are differences in TGF $\beta$ signaling activity between chondrogenic and hypertrophic MSC pellets, we performed Western Blot analysis for the phosphorylated forms of Smad2 and Smad3. The amount of phospho-Smad2 and phospho-Smad3 is clearly reduced in hypertrophic MSC pellets on day 21 and day 28 compared to chondrogenic pellets. The total amount of Smad 2 and Smad3 protein and $\beta$ actin were used as loading control (Fig. 3).

\section{TGF $\beta$ signaling associated genes are down-regulated under hypertrophic conditions}

In order to elucidate a possible role of $\mathrm{TGF} \beta$ signaling
B

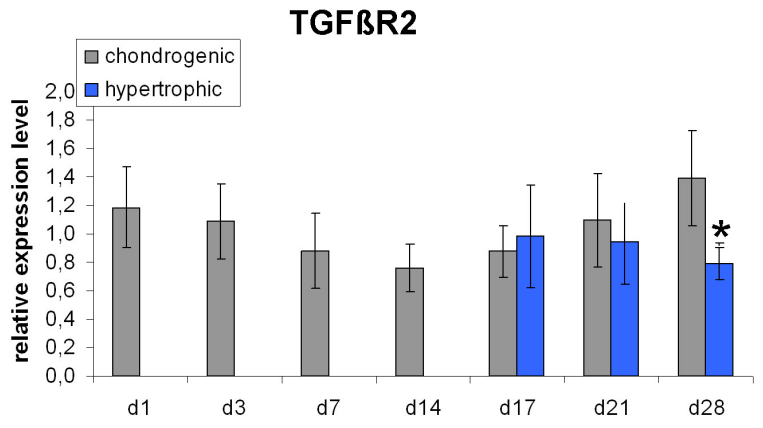

Fig. 2. Gene expression analysis of TGF $\beta$ R1, TGF $\beta$ R2 and Sox9 normalized to HPRT in MSC pellet cultures under chondrogenic and hypertrophy enhancing conditions analyzed by real time $\mathrm{PCR}$. TGF $\beta \mathrm{R} 1$ is significantly down-regulated under hypertrophic conditions on day 17,21 and 28 (A). TGF $\beta$ R2 is down-regulated under hypertrophic conditions on day 28 (B). Sox9 is down-regulated on day 17 and 21 in hypertrophic MSC pellets (C). $n=7$ different donors.

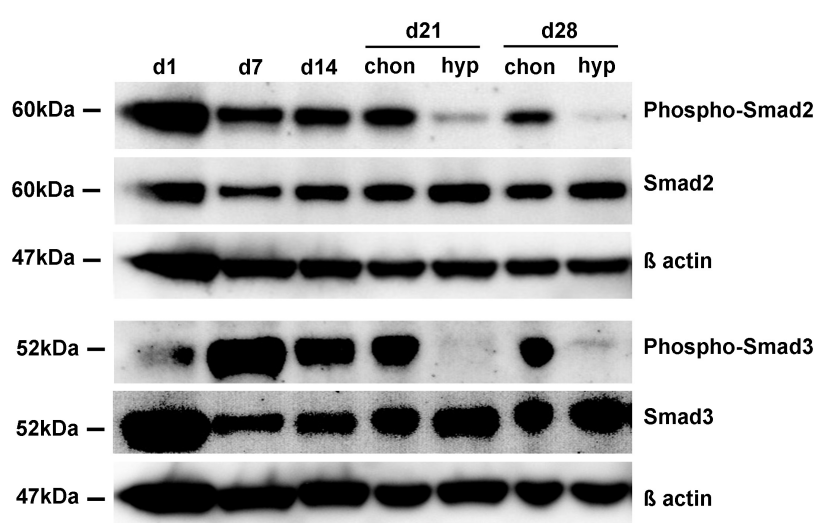

Fig. 3. TGF $\beta$ signaling activity. Western Blot analysis of phospho-Smad2 and phospho-Smad3 under chondrogenic and hypertrophic conditions. The amount of phospho-Smad2 and phospho-Smad3 is reduced under hypertrophic conditions on day 21 and day 28 compared to chondrogenic conditions. The total amount of $\mathrm{Smad} 2 / \mathrm{Smad} 3$ and $\beta$ actin were taken as loading control.

in the regulation of MSC hypertrophy we compared the expression of TGF $\beta$ signaling associated genes between chondrogenic and hypertrophic conditions. The TGF $\beta$ signaling associated transcription factor (via Smad 2/3) Sox 9 is significantly down-regulated on day 17 and day 21 under hypertrophy enhancing conditions. On day 28 
there is a tendency towards decreased Sox 9 expression in the hypertrophic group, without reaching statistical significance (Fig. 2C).

On protein level, Western Blot analysis detected a decreased amount of TGF $\beta \mathrm{R} 1$ protein under hypertrophic conditions on day 21 and day 28 compared to chondrogenic conditions (Fig. 4A). The amount of Sox9 protein is reduced on day 21 under hypertrophic conditions but not on day 28 (Fig. 4B).

\section{The TGF $\beta$-receptor-type-1 inhibitor SB431542}

We investigated the effect of the $\mathrm{TGF} \beta$ antagonist SB431542 on hypertrophic differentiation in the in vitro hMSC hypertrophy model. Under chondrogenic conditions treatment with SB431542 does not enhance hypertrophy. There is no histological difference between MSC
A

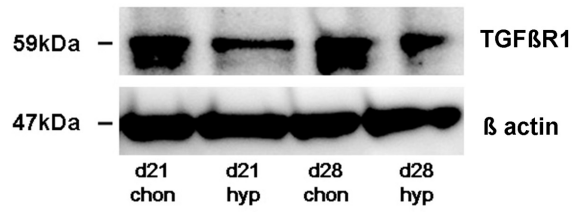

B

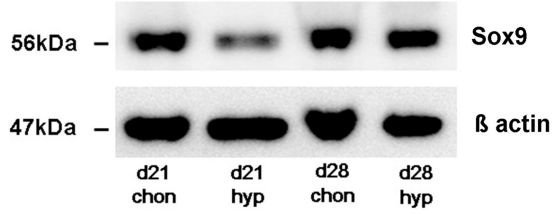

Fig. 4. Western Blot analysis of TGF $\beta \mathrm{R} 1$ and Sox9. TGF $\beta$ R1 protein amount is reduced on day 21 and day 28 under hypertrophic conditions (A). Sox9 protein level is decreased on day 21 under hypertrophic conditions (B). $\beta$ actin was used as loading control.

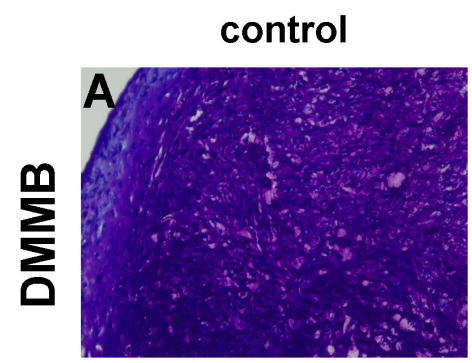

\section{$0,5 \mu M$ SB431542}
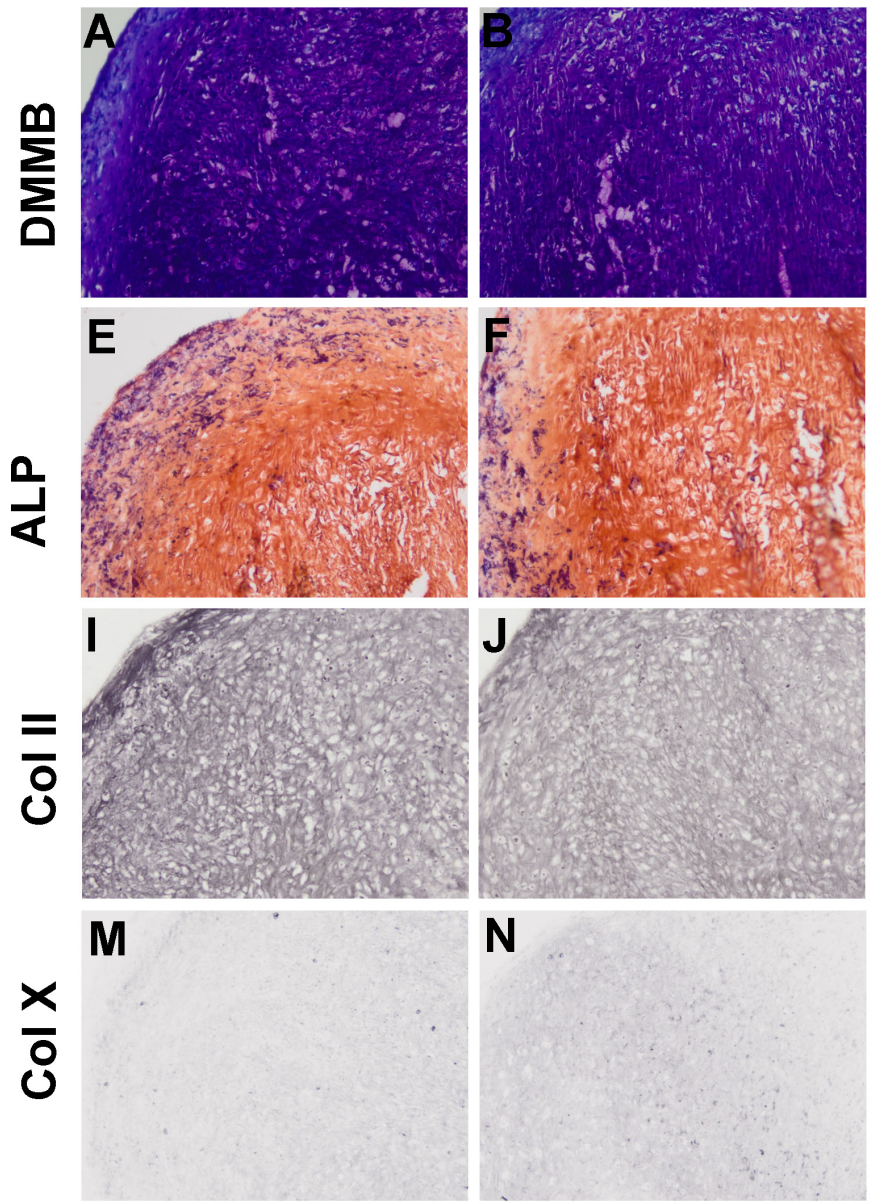
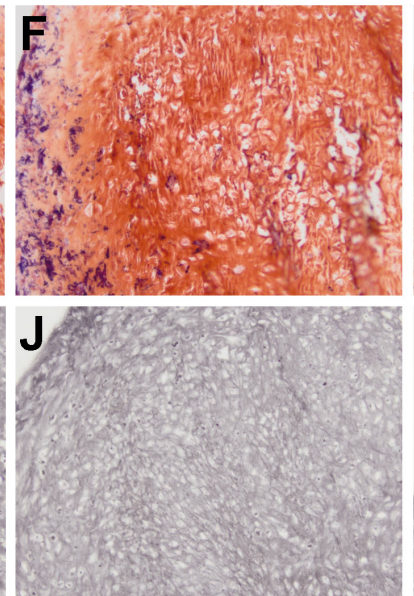

$\mathbf{N}$

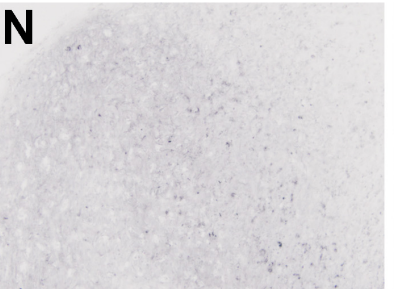

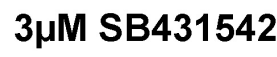
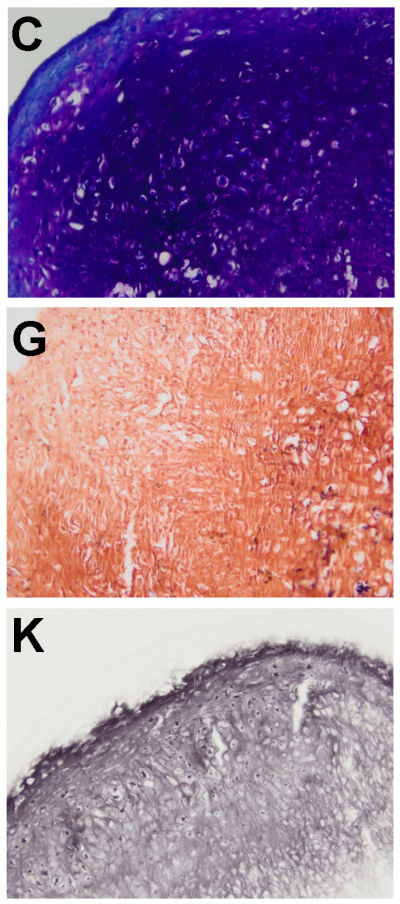

0

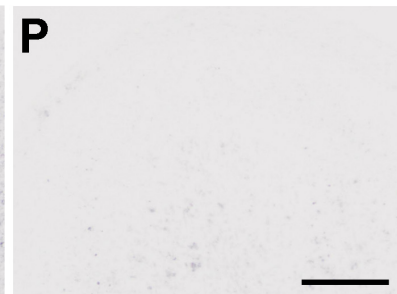

10MM SB431542
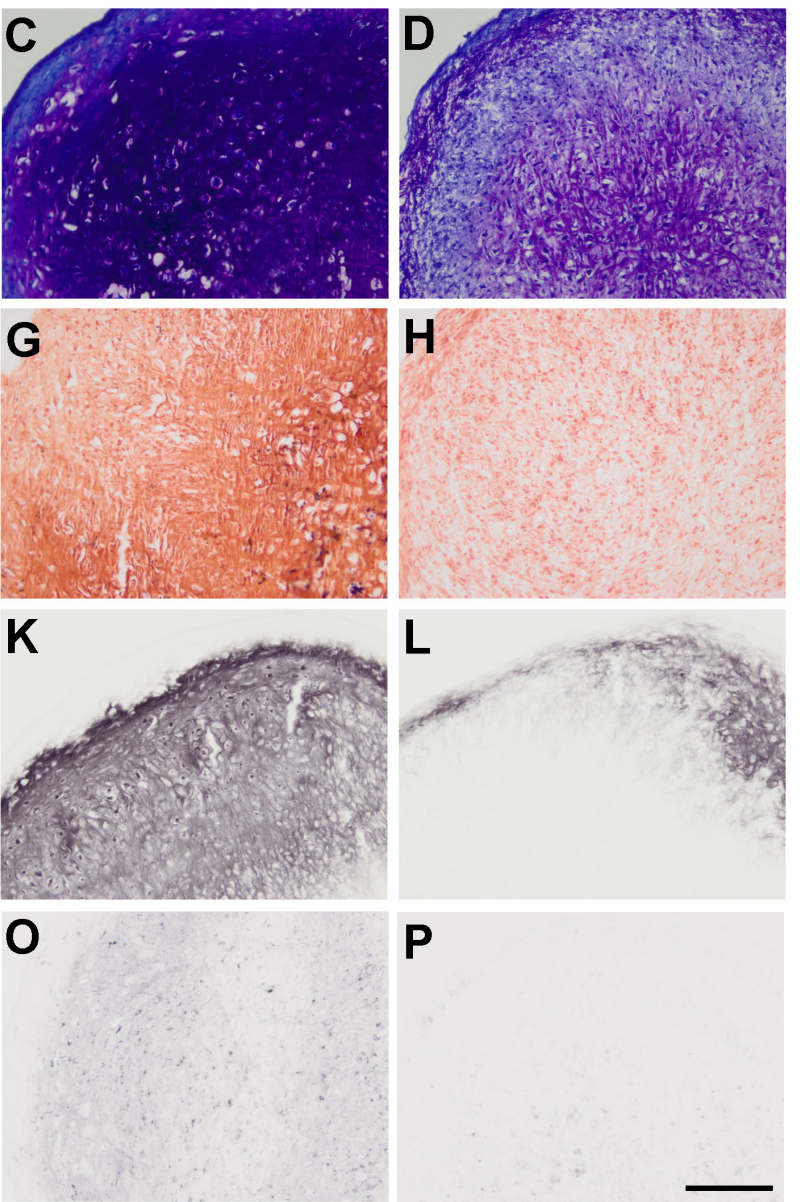

Fig. 5. Histological appearance of MSC pellet cultures on day 28 after SB431542 treatment under chondrogenic conditions. No change in $\mathrm{DMMB}, \mathrm{ALP}$, collagen type II and collagen type $\mathrm{X}$ staining can be detected between chondrogenic control pellets $(A, E, I, M)$ and pellets treated with low doses of SB431542 (B, C, F, G, J, K, N, O). High doses of SB431542 lead to a dedifferentiation of the cells shown by decreased DMMB staining (D) and collagen type II staining (L) (Scale bar $=200 \mu \mathrm{m})$. 
pellets that were treated with low doses of SB431542 (Fig. $5 \mathrm{~B}, \mathrm{C}$ ) and chondrogenic control aggregates (Fig. 5A). Under all conditions a hyaline cartilage like morphology with weak hallmarks of hypertrophy developed. In contrast, high doses of the TGF $\beta$ receptor inhibitor lead to a dedifferentiation of the pellets (Fig. 5D). Similarly, no difference in ALP staining can be detected between chondrogenic control aggregates (Fig. 5E) and SB431542 treated aggregates (Fig. $5 \mathrm{~F} \sim \mathrm{H}$ ). Collagen type II staining is unchanged between chondrogenic control pellets (Fig. 5I) and MSC pellets that were treated with low doses of SB431542 (Fig. 5J, K). However, high doses of the TGF $\beta$ inhibitor strongly reduce Collagen type II staining (Fig. 5L). Collagen type $\mathrm{X}$ staining is weak in chondrogenic control aggregates (Fig. 5M) and in aggregates that were treated with low doses of SB431542 (Fig. 5N, O). In MSC pellets that were treated with $10 \mu \mathrm{M}$ of the TGF $\beta$ inhibitor, no collagen type $\mathrm{X}$ staining can be detected (Fig. 5P).

In the following, we aimed to investigate whether we can increase hypertrophy under chondrogenic conditions by simultaniously inhibiting TGF $\beta$ signaling with SB431542 and addition of bone morphogenetic protein 4 (BMP4). The sole/exclusive addition of BMP4 to chondrogenic medium does not increase the number of hypertrophic cells (Fig. 6A). Simultaneous addition of BMP4 and the TGF $\beta$ inhibitor SB431542 increases the number of hypertrophic cells dose dependently with increasing SB431542 concentration under chondrogenic conditions (Fig. 6B D).

Similarly, there are only few ALP positive cells in chon-
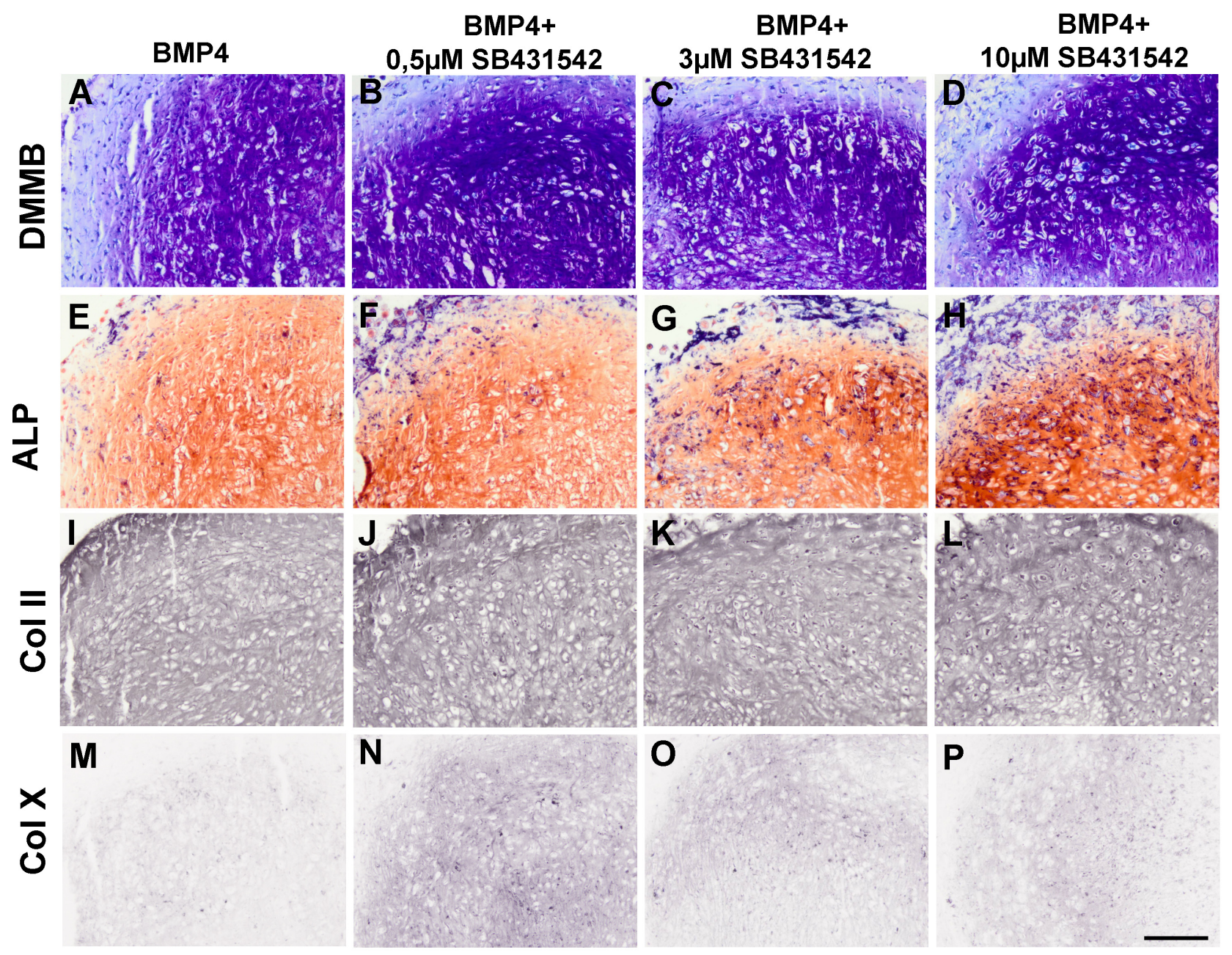

Fig. 6. Histological appearance of MSC pellet cultures on day 28 after SB431542 and BMP4 treatment under chondrogenic conditions. Concomitant application of SB431542 and BMP4 increases hypertrophy in a dose dependent manner shown by an increased amount of hypertrophic $(B \sim D)$ and $A L P$ positive $(F \sim H)$ cells and increased collagen type $X$ staining $(N \sim P)$ after SB431542 and BMP4 treatment, compared to BMP4 only treatment (A, E, M). No difference in collagen type II staining can be detected between BMP4 treated control pellets (I) and pellets treated with SB431542 and BMP4 $(\mathrm{J} \sim \mathrm{L})($ Scale bar $=200 \mu \mathrm{m})$. 
drogenic MSC pellets that were only treated with BMP4 (Fig. 6E). The amount of ALP positive cells increases in chondrogenic MSC pellets that were treated with both, BMP4 and the TGF $\beta$ inhibitor (Fig. $6 \mathrm{~F} \sim \mathrm{H}$ ). The highest amount of ALP positive cells can be detected in pellets that were treated with BMP4 and $10 \mu \mathrm{M}$ SB431542 (Fig. $6 \mathrm{H})$. There is no difference in Collagen type II staining between chondrogenic MSC pellets that were treated with BMP4 (Fig. 6I) and chondrogenic MSC pellets that were treated with BMP4 and the TGF $\beta$ inhibitor (Fig. $6 \mathrm{~J} \sim \mathrm{L}$ ). MSC pellets that were treated with the highest concentration of the TGF $\beta$ inhibitor SB431542 together with BMP4 do not dedifferentiate and show a strong collagen type II staining, indicating that BMP4 preserves chondrogenic differentiation after SB431542 treatment (Fig. 6L). Collagen type X staining is low in chondrogenic MSC pellets that were treated with BMP4 (Fig. 6M). Treatment with BMP4 and SB431542 increases collagen type X staining (Fig. $6 \mathrm{~N} \sim \mathrm{P}$ ) with the highest effects to be shown for lower SB431542 concentrations. However higher concentrations of SB431542 e.g. $3 \mu \mathrm{M}$ and $10 \mu \mathrm{M}$ seem to mitigate the production of collagen type $\mathrm{X}$ within the pellets observed with lower doses e.g. $0.5 \mu \mathrm{M}$ of SB431542.

Under hypertrophic conditions, the TGF $\beta$ inhibitor SB431542 does not further enhance hypertrophy. Nevertheless there are differences detectable between hypertrophic control pellets (Fig. 7A) and pellets that were treated additionally with SB431542 (Fig. 7B D). In a dose dependent manner cells treated with SB431542 under hypertrophic challenge reveal smaller cells with smallest cell sizes observable in the group treated with $0.5 \mu \mathrm{M}$ of TGF $\beta$-receptor-type-1 inhibitor SB431542 whereas large lacunae are visible in the group treated with $10 \mu \mathrm{M}$ of TGF $\beta$-receptor-type-1 inhibitor SB431542 - the highest
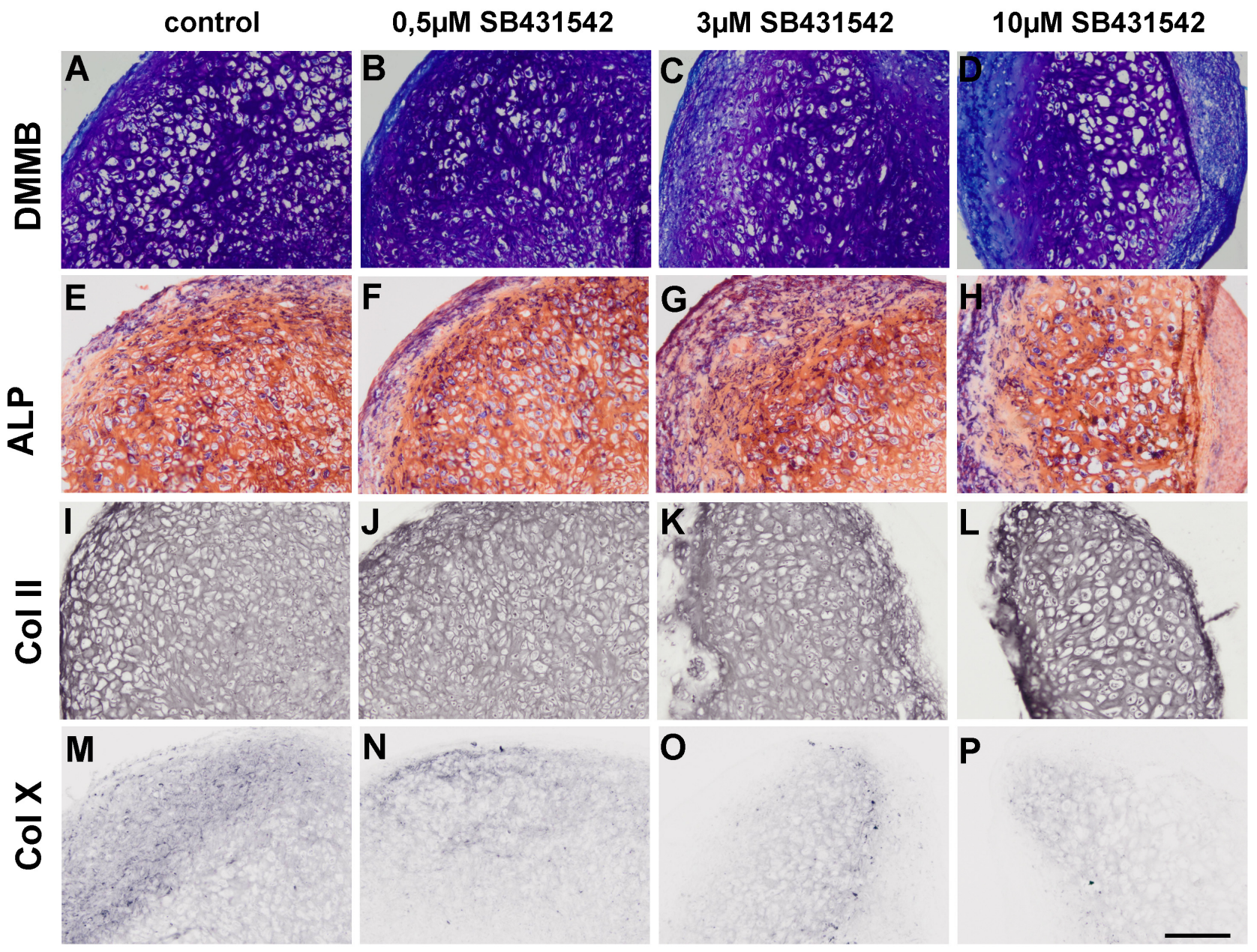

Fig. 7. Histological appearance of MSC pellet cultures on day 28 after SB431542 treatment under hypertrophic conditions. No difference in DMMB, ALP, collagen type II and collagen type $X$ staining can be detected between hypertrophic control pellets (A, E, I, M) and SB431542 treated pellets $(B \sim D, F \sim H, J \sim L, N \sim P)$ (Scale bar $=200 \mu \mathrm{m})$. 
dosage in the present study. After all, cellse still maintain the chondrogenic - but hypertrophic - phenotype, despite increased inhibition of TGF- $\beta$ signaling. Pro-hypertrophic signaling is also still evident in the SB431542treated groups as the amount of ALP positive cells is unchanged between hypertrophic control aggregates (Fig. 7E) and SB431542 treated hypertrophic aggregates (Fig. $7 \mathrm{~F} \sim \mathrm{H}$ ). No difference in collagen type II staining can be detected between the hypertrophic group (Fig. 7I) and the SB431542 treated hypertrophic group (Fig. 7J L). Under hypertrophic control conditions, collagen type $\mathrm{X}$ staining is strong (Fig. 7M). Treatment with $0,5 \mu \mathrm{M}$ and $3 \mu \mathrm{M}$ SB431542 does not change collagen $\mathrm{X}$ staining (Fig. $7 \mathrm{~N}$, O). However, treatment with $10 \mu \mathrm{M} \mathrm{SB} 431542$ and BMP 4 slightly reduces collagen type $\mathrm{X}$ staining (Fig. 7P).

\section{Discussion}

As previously reported (10) hypertrophic conditions were achieved by addition of T3 and withdrawal of dexamethasone after chondrogenic predifferentiation. Similar findings of the present investigation mirror the characteristics of hypertrophic chondrocytes found in the growth plate $(7,10,19)$, allowing to use this hypertrophy model for further analysis.

Furthermore, TGF $\beta$ downstream signaling activity is reduced under hypertrophic conditions. Our findings suggest that this effect is most likely a result of downregulated TGF $\beta$ Receptor expression. These findings are in accordance with a previous study by Serra et al., that showed that transgenic mice, which express a defective TGF $\beta$ Receptor type 2 (TGF $\beta$ R2), have increased hypertrophic differentiation and premature collagen type $\mathrm{X}$ expression in the growth plate (23). The exact mechanism by which hypertrophic conditions reduce the expression of $\mathrm{TGF} \beta$ receptors remains to be elucidated. However, our results suggest that the downregulation of TGF $\beta$-receptors contribute to hypertrophy in consonance with reduced TGF $\beta$ ligands. TGF $\beta$ Rl was shown to be present mainly in resting and hypertrophic chondrocytes within the growth plate and in the perichondrium (25). Further on in situ hybrdization of TGF- $\beta$ receptor expression during development suggested that $\mathrm{TGF} \beta \mathrm{R} 2$ is expressed in chondrogenic condensation centers in embryonal chicken limb buds (26), but not TGF $\beta$ R1. In a mouse model TGF $\beta \mathrm{R} 2$ was detected in developing joints especially in interzone cells (27). In addition to that Spagnoli et al. reported, that $\mathrm{TGF} \beta 2 \mathrm{R}$ conditioned knockouts failed to develop proximal and medial interphalangeal joints, displayed smaller ossification centers and therefore showed reduced limb length (27). After all it has to be mentioned, that complete knockouts of $\mathrm{TGF} \beta \mathrm{R} 2$ are nonviable.

TGF $\beta$ signaling utilizes intracellular Smad proteins to transduce the signal from the activated receptor to the nucleus. Upon activation of the TGF $\beta$ receptor, intracellular Smads2 and Smads3 get phosphorylated and move to the nucleus to activate downstream gene expression. The results of our performed Western Blot analysis for the phosphorylated forms of Smad2 and Smad3 indicate that TGF $\beta$ signaling is less active under hypertrophic conditions. TGF $\beta$ signaling is follows a strict spatio-temporal expression pattern in vivo during enchondral ossification (28) and is downregulated in hypertrophic areas of the growth plate (28). These findinings have been reported in mice (28) and rats (29). Our results show that human hypertrophic MSCs show similar expression patterns of TGF $\beta$ in vitro and therefore it is likely that the above in vivo results may be transferred to human MSC chondrogenesis. Also, Western blotting reinforced our rtPCR results and revealed again suppression of TGF $\beta$ receptors 1 and 2 as well as Sox 9 under hypertrophic conditions. Sox 9 is known to inhibit hypertrophy by inhibiting Runx2 (30) via Nkx3.2 (31). The main reason for downregulation of Sox 9 in the context of hypertrophy is the downregulation of Smad 2/3 phosphorylation due to reduced TGF $\beta$ receptor signaling, orchestrated by lack of the ligand - TGF $\beta-$ and reduction of TGF $\beta \mathrm{R} 1$ and TGF $\beta \mathrm{R} 2$ expression. Our study shows that TGF $\beta$ associated genes are downregulated in hypertrophic conditions.

The experimental data obtained from gene expression analysis and Western blotting showed that TGF $\beta$ receptors are down-regulated and TGF $\beta$ signaling activity is reduced under hypertrophic conditions. We hypothesized that the reduced TGF $\beta$ signaling activity under hypertrophic conditions contributes to the enhancement of hypertrophy. In order to test this, we investigated the effect of the TGF $\beta$ antagonist SB431542 on hypertrophic differentiation in the in vitro hMSC hypertrophy model. SB431542 inhibits TGF $\beta$ R1 kinase activity and thereby inhibits the phosphorylation of $\operatorname{Smad} 3(32,33)$. The inhibition of TGF $\beta$ signaling with SB431542 does not enhance hypertrophy under chondrogenic conditions. During endochondral ossification loss of function models of TGF $\beta$, leading to decreased TGF $\beta$ signaling, increased chondrocyte hypertrophy in vivo $(23,24)$. High doses of the TGF $\beta$ inhibitor lead to a dedifferentiation of the cells shown by decreased metachromatic DMMB staining and decreased collagen type II staining, suggesting a dose dependent inhibition of TGF $\beta$ signaling.

Previous results showed that bone morphogenetic pro- 
tein 4 (BMP4) has strong pro-hypertrophic properties and is able to increase hypertrophy under hypertrophic but not under chondrogenic conditions (34). Adding BMP4 under chrondrogenic conditions presented similar results in this investigation.

We were able to show that the simultaneous addition of the TGF $\beta$ inhibitor and BMP4 increases hypertrophy in hMSCs. This was shown by an increased amount of hypertrophic, ALP positive cells and increased collagen type $\mathrm{X}$ staining. These findings in human MSCs are in accordance with reports by Callahan et al. (33), who found in bone organ cultures of mouse embryos a prohypertrophic effect, when blocking endogenous TGF- $\beta$ stimulation by addition of SB431542 under simultaneous BMP-2 treatment. Further on it was suggested that TGF- $\beta$ signaling induces a negative feedback loop on BMP signaling by Ski related novel protein $\mathrm{N}$ (SnoN), which is reduced upon addition of SB431542 (35). We were able to show that the simultaneous addition of the TGF $\beta$ inhibitor and BMP4 increases hypertrophy in hMSCs. This was proven by an increased amount of hypertrophic, ALP positive cells and increased collagen type $\mathrm{X}$ staining.

Despite the inhibition of TGF $\beta$ sginaling with a high dosage of SB431542, hypertrophy could not be enhanced which gives evidence for the maintainance of the chondrogenic phenotype. This finding suggests that alternative chondrogenic stimulation e.g. via the BMP-signaling pathway is capable of maintaining this differentiation status in vitro. Controversially, loss of function models of TGF $\beta$, leading to decreased TGF $\beta$ signaling, increased chondrocyte hypertrophy in vivo $(22,23)$ during endochondral ossification. The collagen II and X staining under treatment with SB431542 and BMP 4 mirrors the results of SB431542 treatment under purely chondrogenic conditions. Of note is that the cell phenotype was converted dose dependently towards hypertrophy under the regimen off the two prohypertrophic agents BMP 4 (34) and the TGF $\beta$-receptor-type-1 inhibitor SB431542 (32) while hypertrophy in terms of loss of collagen 2 staining and increased collagen 10 production is not evident.

In summary, our results show that TGF $\beta$ signaling is downregulated under hypertrophy enhancing conditions using an established in vitro hypertrophy model in human MSCs. We could show lower TGF $\beta$ receptor expression, reduced Smad-2 and 3 activation and lower expression of downstream molecules. Further the TGF $\beta$ inhibitor SB431542 increases hypertrophy under chondrogenic conditions when applied together with BMP4. However SB431542 is not capable to enhance hypertrophy under chondrogenic conditions without additional BMP-4 treat- ment. Together with previous findings, these results suggest that the enhancement of hypertrophy in this model is a result of both, activation of pro-hypertrophic BMP signalling (32) and reduction of anti-hypertrophic TGF $\beta$ signaling. Thus similar regulatory mechanisms of hypertrophy as in embryonic endochondral ossification plays a role in this in vitro model.

\section{Conclusions}

These experiments showed that the expression of TGF $\beta$-type-1- and 2-receptor and Sox 9 were significantly downregulated upon hypertrophic challenge of the cell constructs as well as phosphorylation of downstream regulators such as Smad 2 and 3 was reduced. Inhibition of TGF $\beta$-receptor-type-1 by SB431542 did not initiate hypertrophy under pro-chondrogenic conditions, but enhanced hypertrophic hallmarks when applied simultaneously with BMP-4.

Overall our results suggest that hypertrophy in this model is enhanced as a result of both activation of pro-hypertrophic BMP signaling and reduction of anti-hypertrophic TGF $\beta$ signaling.

\section{Acknowledgments}

We thank Ms D. Drenkard and Mr. R. Kujat for technical support. This work was funded by DFG grant MU2318/3-1. The authors declare no conflict of interest with the work presented.

\section{Potential Conflict of Interest}

The authors have no conflicting financial interest.

\section{References}

1. Barry F, Boynton RE, Liu B, Murphy JM. Chondrogenic differentiation of mesenchymal stem cells from bone marrow: differentiation-dependent gene expression of matrix components. Exp Cell Res 2001;268:189-200

2. Ichinose S, Tagami M, Muneta T, Sekiya I. Morphological examination during in vitro cartilage formation by human mesenchymal stem cells. Cell Tissue Res 2005;322:217-226

3. Nöth U, Tuli R, Osyczka AM, Danielson KG, Tuan RS. In vitro engineered cartilage constructs produced by presscoating biodegradable polymer with human mesenchymal stem cells. Tissue Eng 2002;8:131-144

4. Sekiya I, Vuoristo JT, Larson BL, Prockop DJ. In vitro cartilage formation by human adult stem cells from bone marrow stroma defines the sequence of cellular and molecular events during chondrogenesis. Proc Natl Acad Sci U S A 2002;99:4397-4402

5. Song L, Baksh D, Tuan RS. Mesenchymal stem cell-based 
cartilage tissue engineering: cells, scaffold and biology. Cytotherapy 2004;6:596-601

6. Johnstone B, Hering TM, Caplan AI, Goldberg VM, Yoo JU. In vitro chondrogenesis of bone marrow-derived mesenchymal progenitor cells. Exp Cell Res 1998;238:265-272

7. Mackay AM, Beck SC, Murphy JM, Barry FP, Chichester $\mathrm{CO}$, Pittenger MF. Chondrogenic differentiation of cultured human mesenchymal stem cells from marrow. Tissue Eng 1998;4:415-428

8. Winter A, Breit S, Parsch D, Benz K, Steck E, Hauner H, Weber RM, Ewerbeck V, Richter W. Cartilage-like gene expression in differentiated human stem cell spheroids: a comparison of bone marrow-derived and adipose tissue-derived stromal cells. Arthritis Rheum 2003;48:418-429

9. Mwale F, Girard-Lauriault PL, Wang HT, Lerouge S, Antoniou J, Wertheimer MR. Suppression of genes related to hypertrophy and osteogenesis in committed human mesenchymal stem cells cultured on novel nitrogen-rich plasma polymer coatings. Tissue Eng 2006;12:2639-2647

10. Mueller MB, Tuan RS. Functional characterization of hypertrophy in chondrogenesis of human mesenchymal stem cells. Arthritis Rheum 2008;58:1377-1388

11. Mueller MB, Fischer M, Zellner J, Berner A, Dienstknecht T, Prantl L, Kujat R, Nerlich M, Tuan RS, Angele P. Hypertrophy in mesenchymal stem cell chondrogenesis: effect of TGF-beta isoforms and chondrogenic conditioning. Cells Tissues Organs 2010;192:158-166

12. Pelttari K, Winter A, Steck E, Goetzke K, Hennig T, Ochs BG, Aigner T, Richter W. Premature induction of hypertrophy during in vitro chondrogenesis of human mesenchymal stem cells correlates with calcification and vascular invasion after ectopic transplantation in SCID mice. Arthritis Rheum 2006;54:3254-3266

13. Scotti C, Tonnarelli B, Papadimitropoulos A, Scherberich A, Schaeren S, Schauerte A, Lopez-Rios J, Zeller R, Barbero A, Martin I. Recapitulation of endochondral bone formation using human adult mesenchymal stem cells as a paradigm for developmental engineering. Proc Natl Acad Sci U S A 2010;107:7251-7256

14. Seyedin SM, Thompson AY, Bentz H, Rosen DM, McPherson JM, Conti A, Siegel NR, Galluppi GR, Piez KA. Cartilage-inducing factor-A. Apparent identity to transforming growth factor-beta. J Biol Chem 1986;261:56935695

15. Schofield JN, Wolpert L. Effect of TGF-beta 1, TGF-beta 2, and bFGF on chick cartilage and muscle cell differentiation. Exp Cell Res 1990;191:144-148

16. Leonard CM, Fuld HM, Frenz DA, Downie SA, Massagué J, Newman SA. Role of transforming growth factor-beta in chondrogenic pattern formation in the embryonic limb: stimulation of mesenchymal condensation and fibronectin gene expression by exogenenous TGF-beta and evidence for endogenous TGF-beta-like activity. Dev Biol 1991;145:99109

17. Joyce ME, Roberts AB, Sporn MB, Bolander ME. Transforming growth factor-beta and the initiation of chondro- genesis and osteogenesis in the rat femur. J Cell Biol 1990; 110:2195-2207

18. Chimal-Monroy J, Díaz de León L. Differential effects of transforming growth factors beta 1 , beta 2 , beta 3 and beta 5 on chondrogenesis in mouse limb bud mesenchymal cells. Int J Dev Biol 1997;41:91-102

19. Ballock RT, Heydemann A, Wakefield LM, Flanders KC, Roberts AB, Sporn MB. TGF-beta 1 prevents hypertrophy of epiphyseal chondrocytes: regulation of gene expression for cartilage matrix proteins and metalloproteases. Dev Biol 1993;158:414-429

20. Böhme K, Winterhalter KH, Bruckner P. Terminal differentiation of chondrocytes in culture is a spontaneous process and is arrested by transforming growth factor-beta 2 and basic fibroblast growth factor in synergy. Exp Cell Res 1995;216:191-198

21. Ferguson CM, Schwarz EM, Reynolds PR, Puzas JE, Rosier $\mathrm{RN}$, O'Keefe RJ. Smad 2 and 3 mediate transforming growth factor-betal-induced inhibition of chondrocyte maturation. Endocrinology 2000;141:4728-4735

22. Ferguson CM, Schwarz EM, Puzas JE, Zuscik MJ, Drissi $\mathrm{H}$, O'Keefe RJ. Transforming growth factor-betal induced alteration of skeletal morphogenesis in vivo. J Orthop Res 2004;22:687-696

23. Serra R, Johnson M, Filvaroff EH, LaBorde J, Sheehan DM, Derynck R, Moses HL. Expression of a truncated, kinase-defective TGF-beta type II receptor in mouse skeletal tissue promotes terminal chondrocyte differentiation and osteoarthritis. J Cell Biol 1997;139:541-552

24. Yang X, Chen L, Xu X, Li C, Huang C, Deng CX. TGF-beta/Smad3 signals repress chondrocyte hypertrophic differentiation and are required for maintaining articular cartilage. J Cell Biol 2001;153:35-46

25. Matsunobu T, Torigoe K, Ishikawa M, de Vega S, Kulkarni $\mathrm{AB}$, Iwamoto Y, Yamada Y. Critical roles of the TGF-beta type I receptor ALK5 in perichondrial formation and function, cartilage integrity, and osteoblast differentiation during growth plate development. Dev Biol 2009;332:325-338

26. Cooley JR, Yatskievych TA, Antin PB. Embryonic expression of the transforming growth factor beta ligand and receptor genes in chicken. Dev Dyn 2014;243:497-508

27. Spagnoli A, O'Rear L, Chandler RL, Granero-Molto F, Mortlock DP, Gorska AE, Weis JA, Longobardi L, Chytil A, Shimer K, Moses HL. TGF-beta signaling is essential for joint morphogenesis. J Cell Biol 2007;177:1105-1117

28. Hayata T, Ezura Y, Asashima M, Nishinakamura R, Noda M. Dullard/Ctdnepl regulates endochondral ossification via suppression of TGF- $\beta$ signaling. J Bone Miner Res 2015;30:318-329

29. Matsunaga S, Yamamoto T, Fukumura K. Temporal and spatial expressions of transforming growth factor-betas and their receptors in epiphyseal growth plate. Int J Oncol 1999;14:1063-1067

30. Dy P, Wang W, Bhattaram P, Wang Q, Wang L, Ballock RT, Lefebvre V. Sox9 directs hypertrophic maturation and blocks osteoblast differentiation of growth plate chondro- 
cytes. Dev Cell 2012;22:597-609

31. Yamashita S, Andoh M, Ueno-Kudoh H, Sato T, Miyaki S, Asahara H. Sox9 directly promotes Bapxl gene expression to repress Runx2 in chondrocytes. Exp Cell Res 2009;315: 2231-2240

32. Inman GJ, Nicolás FJ, Callahan JF, Harling JD, Gaster LM, Reith $\mathrm{AD}$, Laping NJ, Hill CS. SB-431542 is a potent and specific inhibitor of transforming growth factor-beta superfamily type I activin receptor-like kinase (ALK) receptors ALK4, ALK5, and ALK7. Mol Pharmacol 2002;62: 65-74

33. Callahan JF, Burgess JL, Fornwald JA, Gaster LM, Harling JD, Harrington FP, Heer J, Kwon C, Lehr R, Mathur A, Olson BA, Weinstock J, Laping NJ. Identification of novel inhibitors of the transforming growth factor betal (TGFbetal) type 1 receptor (ALK5). J Med Chem 2002;45:9991001

34. Karl A, Olbrich N, Pfeifer C, Berner A, Zellner J, Kujat R, Angele P, Nerlich M, Mueller MB. Thyroid hormone-induced hypertrophy in mesenchymal stem cell chondrogenesis is mediated by bone morphogenetic protein-4. Tissue Eng Part A 2014;20:178-188

35. Kawamura I, Maeda S, Imamura K, Setoguchi T, Yokouchi M, Ishidou Y, Komiya S. SnoN suppresses maturation of chondrocytes by mediating signal cross-talk between transforming growth factor- $\beta$ and bone morphogenetic protein pathways. J Biol Chem 2012;287:29101-29113 